\title{
Semiclassical "Divide-and-Conquer" Method for Spectroscopic Calculations of High Dimensional Molecular Systems
}

\author{
Michele Ceotto, Giovanni Di Liberto, and Riccardo Conte \\ Dipartimento di Chimica, Università degli Studi di Milano, via C. Golgi 19, 20133 Milano, Italy
}

(Received 16 January 2017; published 6 July 2017)

\begin{abstract}
A new semiclassical "divide-and-conquer" method is presented with the aim of demonstrating that quantum dynamics simulations of high dimensional molecular systems are doable. The method is first tested by calculating the quantum vibrational power spectra of water, methane, and benzene-three molecules of increasing dimensionality for which benchmark quantum results are available — and then applied to $\mathrm{C}_{60}$, a system characterized by 174 vibrational degrees of freedom. Results show that the approach can accurately account for quantum anharmonicities, purely quantum features like overtones, and the removal of degeneracy when the molecular symmetry is broken.
\end{abstract}

DOI: 10.1103/PhysRevLett.119.010401

Quantum computational approaches to the spectroscopy of small or medium-sized molecules are very popular. Among them, we recall variational methods like vibrational configuration interaction [1-4] and multiconfiguration time-dependent Hartree [5-7], or perturbative ones such as the second-order vibrational perturbation theory [8-10]. Spectroscopy of high dimensional systems is more difficult to perform since exact quantum simulations are unaffordable and even experimental spectra are often too crowded for an undisputed assignment. A new computationally affordable strategy is needed, while spectra would certainly be much easier to read if they were decomposed into several partial ones.

For this purpose, a novel theoretical approach is presented here. It is based on a semiclassical (SC) "divide-andconquer" strategy that leads to reliable calculations of higher dimensional systems than those ordinarily affordable with quantum methods. Full spectra are regained as a collection of partial ones, quantum effects are included, and a sound spectroscopic interpretation is obtained. This new method fills in the gap between a purely classical spectroscopic study, which is not satisfactory because it neglects key quantum features, and quantum approaches, which often require the setup of a grid of points with a computational cost that exponentially scales with the dimensionality of the system.

In a semiclassical approach [11-43], spectra are calculated in a time-dependent way from classically evolved trajectories, and, if convenient, precomputation of the potential [44-52] can be avoided in favor of a direct dynamics [42,5357], thus allowing one to explore the global potential energy surface also when dealing with high dimensional systems. Recently, we have advanced Miller's pivotal semiclassical initial value representation (SCIVR) [58-63] theory by developing the multiple-coherent (MC) SCIVR approach $[53,64]$. The method exploits pioneering work by De Leon and Heller, which demonstrated that even single-trajectory semiclassical simulations are able to precisely reproduce quantum eigenvalues and eigenfunctions [65]. MC SCIVR is based on a tailored coherent state semiclassical representation and yields highly accurate results in spectroscopy calculations, often within $1 \%$ of the exact result, given a few classical trajectories as input. Applications have faithfully reproduced a variety of quantum effects, including quantum resonances, intramolecular and dipole splitting, and the quantum resonant umbrella inversion in ammonia [66-71]. However, the approach runs out of steam when the dimensionality increases and it is limited to about 20-25 degrees of freedom.

To understand the reasons for such a limitation, we observe that an $N$-dimensional semiclassical wave packet is built as the direct product of monodimensional coherent states $|\chi(t)\rangle=\left|\chi_{1}(t)\right\rangle \ldots\left|\chi_{N}(t)\right\rangle$, and power spectra are obtained by Fourier transforming the recurring timedependent overlap $\langle\chi(0) \mid \chi(t)\rangle$. Consequently, for a precise spectral density, it is essential that the time-evolved semiclassical wave packet significantly overlaps with its initial guess. More specifically, the multidimensional classical trajectory must visit phase space configurations $\left(\mathbf{p}_{t}, \mathbf{q}_{t}\right)$ that are close enough to the starting one $\left(\mathbf{p}_{0}, \mathbf{q}_{0}\right)$. The curse of dimensionality occurs because all of the monodimensional coherent state overlaps $\left[\left\langle\chi_{i}(0) \mid \chi_{i}(t)\right\rangle\right]$ should be sizable almost simultaneously, but, for oscillators with noncommensurable frequencies (even if uncoupled), the concomitant overlapping event is more and more unlikely as the dimensionality increases. The difference between a semiclassical and a classical simulation based on a dipole-dipole correlation function is evident here. In fact, the dipole is always a three-dimensional vector, so it is easier to have a substantial time-dependent overlap.

Figure 1 illustrates how we think to overcome the curse of dimensionality in semiclassical calculations. In a few words, a full-dimensional classical trajectory (the black line) has better odds of getting close to its initial configuration if projected onto a subspace (the red line). Based on 




FIG. 1. Pictorial representation of the projection procedure.

this observation, we propose that, while classical trajectories are treated in full dimensionality, the semiclassical calculation employs subspace bounded information to yield projected spectra. From a statistical point of view, the procedure corresponds to the calculation of a marginal distribution in each subspace after marginalizing out the other degrees of freedom [72]. As a final step, the composition of the several projected spectra provides the full-dimensional one.

We apply this idea to spectral density calculations, $I(E)$ :

$$
I(E) \equiv \frac{1}{2 \pi \hbar} \int_{-\infty}^{+\infty}\left\langle\chi\left|e^{-i \hat{H} t / \hbar}\right| \chi\right\rangle e^{i E t / \hbar} d t .
$$

An exact representation of the quantum propagator $e^{-i \hat{H} t / \hbar}$ is given by Feynman's path integral formulation, which can be approximated by considering only the classical paths connecting points $\mathbf{q}_{0}$ and $\mathbf{q}^{\prime}$ in time $t$ (roots) and including fluctuations up to the second order around the classical action $\left(S^{\mathrm{cl}}\right)$ of each path $[73,74]$,

$$
\left\langle\mathbf{q}^{\prime}\left|e^{-i \hat{H} t / \hbar}\right| \mathbf{q}_{0}\right\rangle \approx \sum_{\text {roots }}\left(\frac{\left|-\frac{\partial^{2} S_{t}^{\mathrm{cl}}}{\partial \mathbf{q}^{\prime} \partial \mathbf{q}_{0}}\right|}{(2 \pi i \hbar)^{N}}\right)^{1 / 2} \frac{e^{i S_{t}^{\mathrm{cl}}\left(\mathbf{q}^{\prime}, \mathbf{q}_{0}\right) / \hbar}}{e^{i v \pi / 2}} .
$$

Equation (2) represents the semiclassical approximation to the Feynman path integral [75]. The term $e^{-i v \pi / 2}$, where $v$ is the integer Maslov index, ensures the continuity of the square root of the preexponential factor. However, the drawback of Eq. (2) is the presence of points at which the determinant in the preexponential factor becomes singular. Miller's SCIVR $[76,77]$ overcomes this issue by replacing the sum over classical trajectories with an integration over initial momenta, a very powerful approach-especially when combined with Heller's coherent state $(|\mathbf{p}, \mathbf{q}\rangle)$ representation. Coherent states have a Gaussian coordinate-space representation whose width is given by the (usually diagonal) $\Gamma$ width matrix

$\langle\mathbf{x} \mid \mathbf{p}, \mathbf{q}\rangle=\left(\frac{\operatorname{det}(\Gamma)}{\pi^{N}}\right)^{1 / 4} e^{-(\mathbf{x}-\mathbf{q})^{T}(\Gamma / 2)(\mathbf{x}-\mathbf{q})+i \mathbf{p}^{T}(\mathbf{x}-\mathbf{q}) / \hbar}$.

By using Miller's SCIVR and by either reformulating the Feynman paths $[78,79]$ or representing the spectral density $I(E)[59]$ in terms of the coherent states of Eq. (3), one gets to the working formula

$$
\begin{aligned}
I(E)= & \frac{1}{2 \pi \hbar} \int_{-\infty}^{+\infty} d t e^{i E t / \hbar} \frac{1}{(2 \pi \hbar)^{N}} \\
& \times \iint d \mathbf{q}_{0} d \mathbf{p}_{0} C_{t}\left(\mathbf{p}_{0}, \mathbf{q}_{0}\right) e^{i S_{t}\left(\mathbf{p}_{0}, \mathbf{q}_{0}\right) / \hbar} \\
& \times\left\langle\chi \mid \mathbf{p}_{t}, \mathbf{q}_{t}\right\rangle\left\langle\mathbf{p}_{0}, \mathbf{q}_{0} \mid \chi\right\rangle,
\end{aligned}
$$

where

$$
C_{t}\left(\mathbf{p}_{0}, \mathbf{q}_{0}\right)=\sqrt{\frac{1}{2}\left|\frac{\partial \mathbf{q}_{t}}{\partial \mathbf{q}_{0}}+\frac{\partial \mathbf{p}_{t}}{\partial \mathbf{p}_{0}}-i \hbar \Gamma \frac{\partial \mathbf{q}_{t}}{\partial \mathbf{p}_{0}}+\frac{i}{\Gamma \hbar} \frac{\partial \mathbf{p}_{t}}{\partial \mathbf{q}_{0}}\right|} .
$$

In order to accelerate the Monte Carlo integration of Eq. (4), it is possible to insert a time averaging filter $(1 / T) \int_{0}^{T} d t$ without loss of accuracy by virtue of Liouville's theorem. Kaledin and Miller [80,81] worked out the following time averaged (TA) version of Eq. (4):

$$
\begin{aligned}
I(E)= & \left(\frac{1}{2 \pi \hbar}\right)^{N} \iint d \mathbf{p}_{0} d \mathbf{q}_{0} \frac{1}{2 \pi \hbar T} \\
& \times\left|\int_{0}^{T} d t e^{i\left[S_{t}\left(\mathbf{p}_{0}, \mathbf{q}_{0}\right)+E t+\phi_{t}\right] / \hbar}\left\langle\boldsymbol{\chi} \mid \mathbf{p}_{t} \mathbf{q}_{t}\right\rangle\right|^{2},
\end{aligned}
$$

where the additional approximation $\phi_{t}=$ phase $\left[C_{t}\left(\mathbf{p}_{0}, \mathbf{q}_{0}\right)\right]$ has been introduced. Equation (6) is now much easier to converge due to its positive-definite integrand, and it has been tested on several molecules [55,67-69,71,80], yielding very accurate results upon evolution of about 1000 trajectories per degree of freedom. The interested reader can find detailed derivations of the above formulas in Ref. [82] (Chap. 10) or in Ref. [83].

To further reduce the computational overhead to just a handful of trajectories, we have recently developed an implementation of Eq. (6) based on two observations. First, accurate eigenvalues can be extracted from a single trajectory whose energy is not necessarily equal to the exact (but unknown) eigenvalue [65]. Second, for each spectroscopic peak, the most contributing trajectories are those that evolve in the proximity of the vibrational peak energy shell [55]. Based on these considerations, we employ a reference state $|\chi\rangle=\sum_{i=1}^{N_{\text {states }}}\left|\mathbf{p}_{\text {eq }}^{i}, \mathbf{q}_{\text {eq }}^{i}\right\rangle$, written as a combination of coherent states placed at the classical phase space points $\left(\mathbf{p}_{\mathrm{eq}}^{i}, \mathbf{q}_{\mathrm{eq}}^{i}\right)$. $\mathbf{q}_{\mathrm{eq}}^{i}$ indicates the equilibrium configuration and $\mathbf{p}_{\mathrm{eq}}^{i}$ the corresponding multidimensional momentum. We set $V\left(\mathbf{q}_{\mathrm{eq}}^{i}\right)=0$, and $\mathbf{p}_{\mathrm{eq}}^{i}$ is chosen to be made of harmonically estimated momenta, i.e., $\left(p_{j, \text { eq }}^{i}\right)^{2} / 2 m=\hbar \omega_{j}\left(n_{j}^{i}+1 / 2\right)$ for the generic $j$ th vibrational mode. The set of $\omega_{j}$ 's is obtained by diagonalizing the Hessian matrix at the equilibrium configuration. In this way, we can approximate Eq. (6) to

$$
\begin{aligned}
I(E)= & \frac{1}{(2 \pi \hbar)^{N}} \frac{\operatorname{Re}}{\pi \hbar T} \sum_{i=1}^{n_{\text {states }}} \mid \int_{0}^{T} d t\left\langle\sum_{i=1}^{n_{\text {states }}} \mathbf{p}_{\text {eq }}^{i}, \mathbf{q}_{\text {eq }}^{i} \mid \mathbf{p}_{t}, \mathbf{q}_{t}\right\rangle \\
& \times\left. e^{i\left[S_{t}\left(\mathbf{p}_{\text {eq }}^{i}, \mathbf{q}_{\mathrm{eq}}^{i}\right)+E t+\phi_{t}\right] / \hbar}\right|^{2},
\end{aligned}
$$


where $n_{\text {states }}$ classical trajectories are evolved from the initial conditions $\left(\mathbf{p}_{\mathrm{eq}}^{i}, \mathbf{q}_{\mathrm{eq}}^{i}\right)$. This approach is called multiple-coherent TA SCIVR (MC SCIVR) (also indicated as MC TA SCIVR). MC SCIVR has been shown to be accurate for systems of complexity up to the glycine molecule (i.e., 24 degrees of freedom) [84].

The main theoretical novelty presented in this Letter is that we reformulate Eq. (6) on the basis of projected-trajectory information. First, the $N$-dimensional phase space is conveniently partitioned, i.e., $(\mathbf{p}, \mathbf{q}) \equiv\left(p_{1}, q_{1}, \ldots, \tilde{p}_{i+1}, \tilde{q}_{i+1}\right.$, $\left.\ldots, \tilde{p}_{i+M}, \tilde{q}_{i+M}, \ldots, p_{N}, q_{N}\right)$, where we have highlighted a generic $M$-dimensional subspace $(M<N)$ with tilde variables $(\tilde{\mathbf{p}}, \tilde{\mathbf{q}})$ (see Fig. 1). For this purpose, an analysis is performed concerning the off-diagonal values of the Hessian matrix averaged over a full-dimensional classical trajectory with harmonic zero-point energy. Off-diagonal terms that are bigger than a threshold value $(\varepsilon)$ correspond to coupled modes and are included in the same subspace. The threshold choice is driven by the trade-off between calculation accuracy and feasibility. On one hand, the smaller the threshold value, the smaller the number of neglected interactions and the more accurate the calculation. On the other, the dimensionality of any projected space should not exceed 20-25 degrees of freedom to permit MC-SCIVR calculations in that subspace. Then, we consider that each vector or matrix appearing in Eq. (6) can be exactly projected into each subspace by means of a singular-value decomposition procedure $\mathbf{A}=\mathbf{U} \mathbf{\Sigma} \mathbf{V}$ [85], and can consequently restrict the phase space integration to $\iint d \tilde{\mathbf{p}}_{0} d \tilde{\mathbf{q}}_{0}$. The $M$ dimensional coherent state becomes

$\left\langle\tilde{\mathbf{x}} \mid \tilde{\mathbf{p}}_{t} \tilde{\mathbf{q}}_{t}\right\rangle=\left(\frac{\operatorname{det}(\tilde{\Gamma})}{\pi^{M}}\right)^{1 / 4} e^{-\left(\tilde{\mathbf{x}}-\tilde{\mathbf{q}}_{t}\right)^{T}(\tilde{\mathbf{\Gamma}} / 2)\left(\tilde{\mathbf{x}}-\tilde{\mathbf{q}}_{t}\right)+i \tilde{\mathbf{p}}_{t}^{T}\left(\tilde{\mathbf{x}}-\tilde{\mathbf{q}}_{t}\right) / \hbar,}$

where $\tilde{\boldsymbol{\Gamma}}=\mathbf{U} \mathbf{U}^{T} \boldsymbol{\Gamma} \mathbf{U}^{T} \mathbf{U}$ is the projected Gaussian width matrix obtained from the singular-value decomposition matrix $\mathbf{U}[86]$. Similarly, $\tilde{C}_{t}$ is obtained by projecting its monodromy matrix components. The remaining term of Eq. (6) to be projected is $S_{t}$. While the projection of the kinetic part of the Lagrangian can be obtained exactly, the potential is generally not separable. In an ideal case, $V_{S}\left(\tilde{\mathbf{q}}_{M}\right)$ would be the potential such that, given the initial conditions $\left(\tilde{\mathbf{p}}_{0}, \tilde{\mathbf{q}}_{0}\right)$, the $M$-dimensional trajectory coincides with the projected one. In such an $M$-dimensional dynamics, the positions in the other degrees of freedom $\left(\mathbf{q}_{N_{\text {vib }}-M}\right)$ are downgraded to parameters. In practice, we fix these parameters at equilibrium positions but introduce an external field $\lambda(t)$ to account for the nonseparability of the potential such that

$$
V_{S}\left(\tilde{\mathbf{q}}_{M}\right)=V\left(\tilde{\mathbf{q}}_{M} ; \mathbf{q}_{N_{\mathrm{vib}}-M}^{\mathrm{eq}}\right)+\lambda(t) .
$$

$\lambda(t)$ is not known a priori and we adopt the following expression, which makes Eq. (9) exact (within a constant) in the separable potential limit
$\lambda(t)=V\left(\tilde{\mathbf{q}}_{M} ; \mathbf{q}_{N_{\text {vib }}-M}\right)-\left[V\left(\tilde{\mathbf{q}}_{M} ; \mathbf{q}_{N_{\text {vib }}-M}^{\mathrm{eq}}\right)+V\left(\tilde{\mathbf{q}}_{M}^{\mathrm{eq}} ; \mathbf{q}_{N_{\text {vib }}-M}\right)\right]$.

Moving to applications, we have first tested the accuracy and effectiveness of our new divide-and-conquer semiclassical initial value representation (DC-SCIVR) approach on three different molecular systems, for which exact vibrational eigenenergies are available in the literature.

Water is a low dimensional but strongly coupled system. Its global three-dimensional vibrational space can be divided into a monodimensional one for the bending mode, plus a bidimensional one for the two stretches. We evolved 3500 classical trajectories on a preexisting potential energy surface [87], each one for a total of 30000 atomic time units. The zero-point energy estimated from the projected spectra is $4606 \mathrm{~cm}^{-1}$, to be compared to the $4631 \mathrm{~cm}^{-1}$ value of a full dimensional semiclassical calculation, and the exact quantum value of $4636 \mathrm{~cm}^{-1}$. DC SCIVR reproduces fundamentals concerning the bending and the asymmetric stretch with excellent accuracy (within $10 \mathrm{~cm}^{-1}$ of the exact quantum results), while the symmetric stretch and the first bending overtone are more off the mark $\left(40 \mathrm{~cm}^{-1}\right)$. Overall, the mean absolute error (MAE) is $23 \mathrm{~cm}^{-1}$. Detailed comparisons can be found in the Supplemental Material [83]. Results for water are a remarkable milestone because of the strong internal vibrational coupling of this molecule. In fact, in higher dimensional systems, intermode couplings are generally weaker and DC SCIVR (being exact for separable systems) is expected to perform better once strongly coupled modes are confined in the same subspace.

Another known issue for SC methods comes from chaotic trajectories which can spoil the SC simulation and are, therefore, usually discarded. In an application of DC SCIVR to methane, the nine-dimensional vibrational space has been partitioned into a six-dimensional and a threedimensional one, and it turns out that methane dynamics is highly chaotic with strong quantum effects, given the light mass of the hydrogen atoms. In fact, 95\% of the 180000

TABLE I. Vibrational frequencies of $\mathrm{CH}_{4}$. QM labels the exact quantum eigenvalues, SCIVR refers to a full dimensional semiclassical calculation, DC SCIVR labels frequencies obtained with the divide-and-conquer approach here presented, and HO indicates harmonic estimates. MAE stands for Mean Absolute Error. All values are in $\mathrm{cm}^{-1}$.

\begin{tabular}{lcccr}
\hline \hline State & QM $^{[88]}$ & SCIVR & DC SCIVR & HO \\
\hline $1_{1}$ & 1313 & 1300 & 1300 & 1345 \\
$2_{1}$ & 1535 & 1529 & 1532 & 1570 \\
$1_{2}$ & 2624 & 2594 & 2606 & 2690 \\
$1_{1} 2_{1}$ & 2836 & 2825 & 2834 & 2915 \\
$3_{1}$ & 2949 & 2948 & 2964 & 3036 \\
$2_{2}$ & 3067 & 3048 & 3050 & 3140 \\
$4_{1}$ & 3053 & 3048 & 3044 & 3157 \\
MAE & & 12 & 11 & 68 \\
\hline \hline
\end{tabular}


TABLE II. Comparison between DC SCIVR and the available quantum results (EQD) for benzene fundamental frequencies. Degenerate frequencies are not replicated. Values are in $\mathrm{cm}^{-1}$.

\begin{tabular}{lccccc}
\hline \hline State & DC SCIVR & EQD $^{[90]}$ & State & DC SCIVR & EQD $^{[90]}$ \\
\hline $1_{1}$ & 388 & 399.4554 & $10_{1}$ & 1024 & 1040.98 \\
$2_{1}$ & 610 & 611.4227 & $11_{1}$ & 1157 & 1147.751 \\
$3_{1}$ & 732 & 666.9294 & $12_{1}$ & 1157 & 1180.374 \\
$4_{1}$ & 706 & 710.7318 & $13_{1}$ & 1295 & 1315.612 \\
$5_{1}$ & 908 & 868.9106 & $14_{1}$ & 1357 & 1352.563 \\
$6_{1}$ & 990 & 964.0127 & $15_{1}$ & 1460 & 1496.231 \\
$7_{1}$ & 996 & 985.8294 & $16_{1}$ & 1606 & 1614.455 \\
$8_{1}$ & 996 & 997.6235 & & & \\
$9_{1}$ & 1018 & 1015.64 & MAE & 19 & \\
\hline \hline
\end{tabular}

trajectories run (each one evolved for 30000 atomic time units) has been discarded on the basis of the monodromy determinant conservation criterion [40,81]. Table I provides a comparison between our DC-SCIVR estimates and exact values by Carter et al. on the same analytical surface [88]. This test permits us to show that DC SCIVR works fairly well, with fundamentals and overtones reliably detected and a tiny $\operatorname{MAE}\left(11 \mathrm{~cm}^{-1}\right)$.

Recently, by employing a preexisting potential energy surface [89], Halverson and Poirier calculated a set of quantum vibrational frequencies of benzene with their exact quantum dynamics (EQD) method [90], which we use to benchmark our DC-SCIVR results for this high dimensional molecular system. For this purpose, the vibrational space of benzene has been divided into a larger eight-dimensional subspace plus eight bidimensional and six monodimensional ones. We have evolved 1000 trajectories per degree of freedom for a total of 30000 atomic time units each. Furthermore, an accurate second-order iterative approximation to the preexponential factor $\exp \left[i \phi_{t} / \hbar\right]$, as described in Ref. [71], has been employed to avoid a discarding of chaotic trajectories. Results are reported in Table II and permit us to assess DC-SCIVR accuracy in this challenging application. Even in the case of benzene, DC SCIVR is characterized by a small MAE value $\left(19 \mathrm{~cm}^{-1}\right)$. This is the result of a large majority of highly accurate frequencies and a single mode with lower precision.

Finally, after having benchmarked the accuracy of our method against exact quantum results for three molecules of different dimensionality and complexity, we demonstrate the applicability of DC SCIVR to an extremely high dimensional problem by computing the power spectrum of a fullerenelike system. $\mathrm{C}_{60}$ has 174 vibrational degrees of freedom, a number which makes a fully quantum mechanical calculation as well as a standard semiclassical simulation clearly unfeasible and calls for an efficient alternative method. We employed a preexisting force field derived from density functional theory calculations on graphene sheets. This force field takes into account stretching, bending, and torsional contributions but neglects

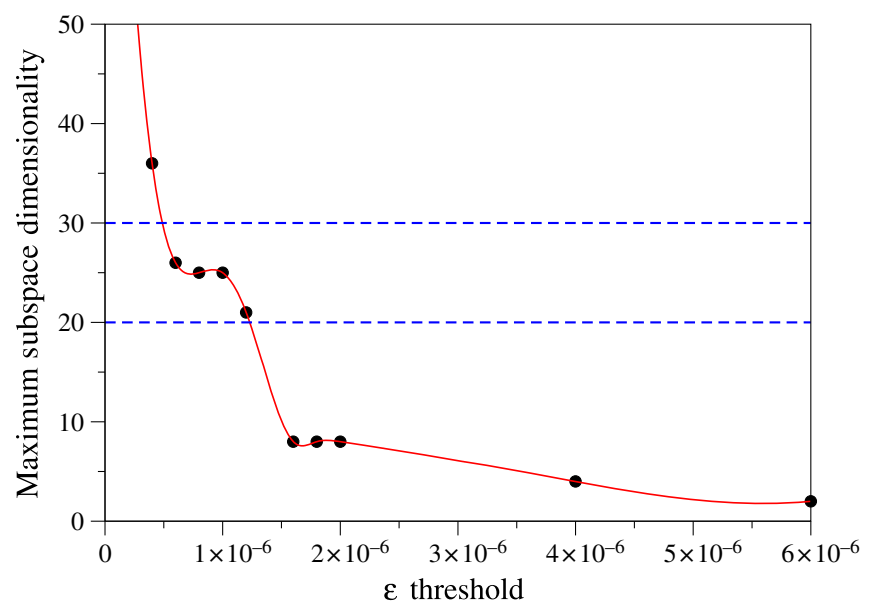

FIG. 2. Maximum subspace dimensionality vs threshold $\varepsilon$ for the $\mathrm{C}_{60}$ calculation. The red curve fits the overall behavior, while the dashed blue lines define the range of desired maximum subspace dimensionality.

bond-coupling terms and van der Waals interactions [91]. It is therefore not tailored on a real fullerene molecule, but the main intent of this final application is to show that our method can overcome the "curse of dimensionality" even in very challenging instances. DC SCIVR starts off with the definition of the subspaces in which the projected spectra must be computed. Figure 2 shows how the choice of the threshold influences the maximum subspace dimensionality for this system. As previously anticipated, a trade-off leads to considering only instances within the dashed blue lines. On the basis of Fig. 2, we have chosen a threshold value of $10^{-6}$, which corresponds to a maximum subspace dimensionality equal to 25 . This choice has permitted us to divide the 174dimensional vibrational space into 90 monodimensional, 1 bidimensional, 3 three-dimensional, 2 six-dimensional, 1



FIG. 3. DC-SCIVR (black line) and classical (red line) spectra for one of the subspaces employed in the $\mathrm{C}_{60}$ calculation. Harmonic frequencies are reported in dashed blue lines. Labels are set according to Table III. 
TABLE III. Frequencies of the $\mathrm{C}_{60}$ model up to $1600 \mathrm{~cm}^{-1}$. HO indicates harmonic values, $\mathrm{Cl}$ labels the classical estimates of fundamental frequencies, and DC SCIVR introduces our semiclassical results. Values are in $\mathrm{cm}^{-1}$.

\begin{tabular}{|c|c|c|c|c|c|c|c|c|c|c|c|c|c|c|c|c|c|c|c|}
\hline State & $\mathrm{HO}$ & $\mathrm{Cl}$ & $\begin{array}{c}\text { DC } \\
\text { SCIVR }\end{array}$ & St. & $\mathrm{HO}$ & $\mathrm{Cl}$ & $\begin{array}{c}\text { DC } \\
\text { SCIVR }\end{array}$ & St. & $\mathrm{HO}$ & $\mathrm{Cl}$ & $\begin{array}{c}\text { DC } \\
\text { SCIVR }\end{array}$ & St. & $\mathrm{HO}$ & $\mathrm{Cl}$ & $\begin{array}{c}\text { DC } \\
\text { SCIVR }\end{array}$ & St. & $\mathrm{HO}$ & $\mathrm{Cl}$ & $\begin{array}{c}\text { DC } \\
\text { SCIVR } \\
\end{array}$ \\
\hline 1 & 255 & 54 & 25 & $10_{1}$ & 68 & 11 & 6 & $5_{2}$ & 808 & & 8 & $23_{1}$ & 14 & 1015 & 10 & $29_{1}$ & 1310 & 1269 & 1264 \\
\hline $2_{1}$ & 318 & 355 & 352 & $11_{1}$ & 601 & 571 & 572 & $17_{1}$ & 816 & 779 & 774 & $24_{1}$ & 1042 & 1039 & 1037 & $13_{2}$ & 1314 & & 1303 \\
\hline $3_{1}$ & 359 & 347 & 346 & $2_{2}$ & 636 & & 706 & $18_{1}$ & 863 & 872 & 872 & $25_{1}$ & 1052 & 1075 & 1075 & $31_{1}$ & 1457 & 1438 & 1434 \\
\hline $4_{1}$ & 404 & 432 & 432 & $12_{1}$ & 648 & 630 & 626 & $19_{1}$ & 890 & 911 & 911 & $26_{1}$ & 1091 & 1062 & 1060 & $32_{1}$ & 1470 & 1398 & 1391 \\
\hline $5_{1}$ & 404 & 404 & 403 & $13_{1}$ & 657 & 652 & 651 & $20_{1}$ & 905 & 880 & 880 & $9_{2}$ & 1092 & & 1091 & $33_{1}$ & 1526 & 1467 & 1506 \\
\hline $6_{1}$ & 484 & 483 & 483 & $3_{2}$ & 718 & & 693 & $21_{1}$ & 962 & 971 & 971 & $10_{2}$ & 1136 & & 1220 & $14_{2}$ & 1540 & & 1534 \\
\hline $7_{1}$ & 488 & 547 & 547 & $14_{1}$ & 770 & 767 & 767 & $6_{2}$ & 968 & & 966 & $11_{2}$ & 1202 & & 1150 & $15_{2}$ & 1550 & & 1533 \\
\hline $8_{1}$ & 494 & 478 & 478 & $15_{1}$ & 775 & 766 & 766 & $7_{2}$ & 976 & & 1093 & $27_{1}$ & 1225 & 1218 & 1218 & $16_{2}$ & 1562 & & 1554 \\
\hline $1_{2}$ & 510 & & 506 & $16_{1}$ & 781 & 777 & 777 & $8_{2}$ & 988 & & 957 & $28_{1}$ & 1252 & 1231 & 1231 & & & & \\
\hline $9_{1}$ & 546 & 545 & 546 & $4_{2}$ & 808 & & 863 & $22_{1}$ & 1000 & 997 & 998 & $12_{2}$ & 1296 & & 1254 & & & & \\
\hline
\end{tabular}

eight-dimensional, two 14-dimensional, and one 25dimensional subspaces. To calculate the projected spectra, we ran 175 classical trajectories, each one evolved for 50000 atomic time units. We employed a reference state $|\chi\rangle$ selected in agreement with the previously described MC-SCIVR recipe and, as in the case of benzene, a second-order iterative approximation to the preexponential factor [71]. Figure 3 reports, as an example, the DC-SCIVR spectrum of one of the subspaces. We have also simulated and plotted a transient full dimensional classical spectrum on the basis of the same trajectories employed for the semiclassical calculations. To better compare the two different simulations, we have shifted the DC-SCIVR spectrum in such a way that the zero-point energy is set to zero. From the comparison, we note that the DC-SCIVR and classical estimates are close to each other. However, DC SCIVR is able to increase the level of knowledge by also detecting quantum overtones. Results up to an energy of about $1600 \mathrm{~cm}^{-1}$ relative to the zero-point energy can be found in Table III.

A concern that may arise about the approach regards its efficiency when dealing with lower-symmetry molecules. Thus, to demonstrate that reduced symmetry is not a hindrance to our calculations, we have investigated an ad hoc constructed fullerene isotope model for which symmetry has been broken. Substitution of three appropriate carbon nuclei with nuclei having the same mass as gold ones removed the degeneracies of the vibrational levels. This model was built to preserve the original nuclear and electronic charges so that the force field would not need to be modified. The result of the isotopic substitution is that previously degenerate frequencies are split already at the harmonic level. Even if such splittings are mostly within semiclassical accuracy (i.e., 25-30 $\mathrm{cm}^{-1}$ ), DC-SCIVR results are resolved enough to detect a multiple-peak feature in the isotopic model opposite to the original case characterized by a lonely (degenerate) peak. A relevant example of this is reported in the Supplemental Material [83].

In summary, we have presented a new approach to the calculation of theoretical vibrational spectra of high dimensional molecular systems. The method has been tested for the small and highly intermode coupled water molecule, the highly chaotic methane molecule, and the high dimensional benzene molecule yielding, in all cases, accurate estimates if compared to the available exact quantum results. Then, it has been demonstrated that application to a sizable system made of 174 degrees of freedom yields an accurate quantum estimate of fundamental and overtone frequencies, thus opening up the possibility of quantum investigating the spectroscopy of highly dimensional systems.

We acknowledge financial support from the European Research Council (ERC) under the European Union's Horizon 2020 research and innovation program (Grant Agreement No. [647107]-SEMICOMPLEX-ERC-2014$\mathrm{CoG})$. The CINECA Italian supercomputing center is thanked for allocating computational time under projects LISA-GREENTI and ISCRAC-VIBROGLY. Professor Bill Poirier is warmly thanked for having provided the benzene potential energy surface and his EQD results.

*michele.ceotto@unimi.it

[1] J. M. Bowman, S. Carter, and X. Huang, Int. Rev. Phys. Chem. 22, 533 (2003).

[2] A. K. Samanta, Y. Wang, J. S. Mancini, J. M. Bowman, and H. Reisler, Chem. Rev. 116, 4913 (2016).

[3] C. Qu, R. Conte, P. L. Houston, and J. M. Bowman, Phys. Chem. Chem. Phys. 17, 8172 (2015).

[4] G. Avila and T. Carrington, Jr., J. Chem. Phys. 135, 064101 (2011).

[5] U. Manthe, J. Theor. Comput. Chem. 1, 153 (2002).

[6] H.-D. Meyer and G. A. Worth, Theor. Chem. Acc. 109, 251 (2003).

[7] J. M. Bowman, T. Carrington, and H.-D. Meyer, Mol. Phys. 106, 2145 (2008).

[8] O. Bludsky, J. Chocholousova, J. Vacek, F. Huisken, and P. Hobza, J. Chem. Phys. 113, 4629 (2000).

[9] V. Barone, J. Chem. Phys. 122, 014108 (2005). 
[10] M. Biczysko, J. Bloino, I. Carnimeo, P. Panek, and V. Barone, J. Mol. Struct. 1009, 74 (2012).

[11] Y. Elran and K. Kay, J. Chem. Phys. 110, 3653 (1999).

[12] D. H. Zhang and E. Pollak, Phys. Rev. Lett. 93, 140401 (2004).

[13] K. G. Kay, Chem. Phys. 322, 3 (2006).

[14] R. Conte and E. Pollak, Phys. Rev. E 81, 036704 (2010).

[15] S. Bonella, G. Ciccotti, and R. Kapral, Chem. Phys. Lett. 484, 399 (2010).

[16] M. Monteferrante, S. Bonella, and G. Ciccotti, J. Chem. Phys. 138, 054118 (2013).

[17] R. Conte and E. Pollak, J. Chem. Phys. 136, 094101 (2012).

[18] J. Petersen and E. Pollak, J. Chem. Phys. 143, 224114 (2015).

[19] D. V. Shalashilin and M. S. Child, J. Chem. Phys. 115, 5367 (2001).

[20] D. V. Shalashilin and M. S. Child, Chem. Phys. 304, 103 (2004).

[21] E. M. Heatwole and O. V. Prezhdo, J. Chem. Phys. 130, 244111 (2009).

[22] S. Garashchuk, V. Rassolov, and O. Prezhdo, Rev. Comput. Chem. 27, 287 (2011).

[23] P. Huo and D. F. Coker, Mol. Phys. 110, 1035 (2012).

[24] H. Pal, M. Vyas, and S. Tomsovic, Phys. Rev. E 93, 012213 (2016)

[25] W. Koch and D. J. Tannor, arXiv:1701.01378.

[26] C. Harabati, J. M. Rost, and F. Grossmann, J. Chem. Phys. 120, 26 (2004).

[27] F. Grossmann, Comments At. Mol. Phys. 34, 141 (1999).

[28] H. Nakamura, S. Nanbu, Y. Teranishi, and A. Ohta, Phys. Chem. Chem. Phys. 18, 11972 (2016).

[29] A. D. Kondorskiy and S. Nanbu, J. Chem. Phys. 143, 114103 (2015).

[30] G. Tao, Theor. Chem. Acc. 133, 1448 (2014).

[31] S. V. Antipov, Z. Ye, and N. Ananth, J. Chem. Phys. 142, 184102 (2015).

[32] J. Liu and W. H. Miller, J. Chem. Phys. 125, 224104 (2006).

[33] J. Liu and W. H. Miller, J. Chem. Phys. 127, 114506 (2007).

[34] J. Liu and W. H. Miller, J. Chem. Phys. 126, 234110 (2007).

[35] J. Liu and W. H. Miller, J. Chem. Phys. 128, 144511 (2008).

[36] S.-I. Koda, J. Chem. Phys. 143, 244110 (2015).

[37] S.-I. Koda, J. Chem. Phys. 144, 154108 (2016).

[38] H. Ushiyama and K. Takatsuka, J. Chem. Phys. 122, 224112 (2005).

[39] S. Takahashi and K. Takatsuka, J. Chem. Phys. 127, 084112 (2007).

[40] Y. Zhuang, M. R. Siebert, W. L. Hase, K. G. Kay, and M. Ceotto, J. Chem. Theory Comput. 9, 54 (2013).

[41] M. Wehrle, M. Sulc, and J. Vanicek, J. Chem. Phys. 140, 244114 (2014).

[42] M. Wehrle, S. Oberli, and J. Vaníček, J. Phys. Chem. A 119, 5685 (2015).

[43] E. Zambrano, M. Šulc, and J. Vaníček, J. Chem. Phys. 139, 054109 (2013).

[44] B. J. Braams and J. M. Bowman, Int. Rev. Phys. Chem. 28, 577 (2009).

[45] R. Conte, B. Fu, E. Kamarchik, and J. M. Bowman, J. Chem. Phys. 139, 044104 (2013).

[46] B. Jiang and H. Guo, J. Chem. Phys. 141, 034109 (2014).
[47] R. Conte, P. L. Houston, and J. M. Bowman, J. Chem. Phys. 140, 151101 (2014).

[48] P. L. Houston, R. Conte, and J. M. Bowman, J. Phys. Chem. A 118, 7758 (2014).

[49] R. Conte, P. L. Houston, and J. M. Bowman, J. Phys. Chem. A 119, 12304 (2015).

[50] R. Conte, C. Qu, and J. M. Bowman, J. Chem. Theory Comput. 11, 1631 (2015).

[51] P. L. Houston, R. Conte, and J. M. Bowman, J. Phys. Chem. A 119, 4695 (2015).

[52] P. L. Houston, R. Conte, and J. M. Bowman, J. Phys. Chem. A 120, 5103 (2016).

[53] M. Ceotto, S. Atahan, G. F. Tantardini, and A. AspuruGuzik, J. Chem. Phys. 130, 234113 (2009).

[54] J. Tatchen and E. Pollak, J. Chem. Phys. 130, 041103 (2009).

[55] M. Ceotto, G. F. Tantardini, and A. Aspuru-Guzik, J. Chem. Phys. 135, 214108 (2011).

[56] S. Y. Y. Wong, D. M. Benoit, M. Lewerenz, A. Brown, and P.-N. Roy, J. Chem. Phys. 134, 094110 (2011).

[57] M. Ceotto, Y. Zhuang, and W. L. Hase, J. Chem. Phys. 138, 054116 (2013).

[58] E. J. Heller, J. Chem. Phys. 75, 2923 (1981).

[59] M. F. Herman and E. Kluk, Chem. Phys. 91, 27 (1984).

[60] W. H. Miller, Proc. Natl. Acad. Sci. U.S.A. 102, 6660 (2005).

[61] K. G. Kay, Annu. Rev. Phys. Chem. 56, 255 (2005).

[62] W. H. Miller, J. Chem. Phys. 53, 1949 (1970).

[63] W. H. Miller, Adv. Chem. Phys. 25, 69 (1974).

[64] M. Ceotto, D. Dell'Angelo, and G. F. Tantardini, J. Chem. Phys. 133, 054701 (2010).

[65] N. De Leon and E. J. Heller, J. Chem. Phys. 78, 4005 (1983).

[66] M. Ceotto, S. Atahan, S. Shim, G. F. Tantardini, and A. Aspuru-Guzik, Phys. Chem. Chem. Phys. 11, 3861 (2009).

[67] M. Ceotto, S. Valleau, G. F. Tantardini, and A. AspuruGuzik, J. Chem. Phys. 134, 234103 (2011).

[68] R. Conte, A. Aspuru-Guzik, and M. Ceotto, J. Phys. Chem. Lett. 4, 3407 (2013).

[69] D. Tamascelli, F. S. Dambrosio, R. Conte, and M. Ceotto, J. Chem. Phys. 140, 174109 (2014).

[70] M. Buchholz, F. Grossmann, and M. Ceotto, J. Chem. Phys. 144, 094102 (2016).

[71] G. Di Liberto and M. Ceotto, J. Chem. Phys. 145, 144107 (2016).

[72] R. J. Trumpler and H. F. Weaver, Statistical Astronomy (Dover Publications, New York, 1962).

[73] M. C. Gutzwiller, J. Math. Phys. (N.Y.) 8, 1979 (1967).

[74] J. H. Van Vleck, Proc. Natl. Acad. Sci. U.S.A., 14, 178 (1928).

[75] M. V. Berry and K. Mount, Rep. Prog. Phys. 35, 315 (1972).

[76] W. H. Miller, J. Chem. Phys. 53, 3578 (1970).

[77] E. J. Heller, J. Chem. Phys. 94, 2723 (1991).

[78] Y. Weissman, J. Chem. Phys. 76, 4067 (1982).

[79] M. Baranger, M. A. de Aguiar, F. Keck, H.-J. Korsch, and B. Schellhaass, J. Phys. A 34, 7227 (2001).

[80] A. L. Kaledin and W. H. Miller, J. Chem. Phys. 119, 3078 (2003). 
[81] A. L. Kaledin and W. H. Miller, J. Chem. Phys. 118, 7174 (2003).

[82] D. J. Tannor, Introduction to Quantum Mechanics (University Science Books, Sausalito, CA, 2007).

[83] See Supplemental Material at http://link.aps.org/ supplemental/10.1103/PhysRevLett.119.010401 for a detailed derivation of formulas (1)-(6).

[84] F. Gabas, R. Conte, and M. Ceotto, J. Chem. Theory Comput. 13, 2378 (2017).

[85] K. Hinsen and G. R. Kneller, Mol. Simul. 23, 275 (2000).

[86] B. B. Harland and P.-N. Roy, J. Chem. Phys. 118, 4791 (2003).
[87] J. M. Bowman, A. Wierzbicki, and J. Zuniga, Chem. Phys. Lett. 150, 269 (1988).

[88] S. Carter, H. M. Shnider, and J. M. Bowman, J. Chem. Phys. 110, 8417 (1999).

[89] P. E. Maslen, N. C. Handy, R. D. Amos, and D. Jayatilaka, J. Chem. Phys. 97, 4233 (1992).

[90] T. Halverson and B. Poirier, J. Phys. Chem. A 119, 12417 (2015).

[91] D. Holec, M. A. Hartmann, F. D. Fischer, F. G. Rammerstorfer, P. H. Mayrhofer, and O. Paris, Phys. Rev. B 81, 235403 (2010). 